\title{
What to do with an equivocal myocardial perfusion study?
}

\author{
Gregory S. Thomas, MD, MPH ${ }^{\mathrm{a}, \mathrm{b}}$
}

See related article, pp. 701-713

The "hedge" - the bane of nuclear medicine testing. Abidov et al provide us with an important contribution with their thoughtful study evaluating the use of CT coronary angiography following "inconclusive" MPI studies.

Prior to delving into what to do with an equivocal MPI, the best option is to avoid an equivocal scan in the first place. How might we accomplish this:

\section{Imaging techniques:}

Motion correction software ${ }^{2}$

Attenuation correction ${ }^{3,4}$

Perform a second image in selected patients

Image prone to allow another view of the inferior wall or the opportunity to see an anterior wall defect shift position as the breast shifts position ${ }^{5}$

Delayed imaging - if the gut overlaps the left ventricle on the initial scan wait for it to clear and repeat the image ${ }^{6}$

Protocols:

Achieve an adequate heart rate or change to pharmacologic stress 6,7

Add low level exercise to pharmacologic stress ${ }^{8,9}$

\section{Clinical information:}

Read the study blind initially but then incorporate stress test and clinical information ${ }^{10}$

From the Mission Internal Medicine Group, ${ }^{\mathrm{a}}$ Mission Viejo, CA; Division of Cardiology, ${ }^{\mathrm{b}}$ University of California, Irvine, Orange, CA.

Reprint requests: Gregory S. Thomas, MD, MPH, Mission Internal Medicine Group, Mission Viejo, CA; gthomas9@mac.com.

J Nucl Cardiol 2009;16:683-5.

$1071-3581 / \$ 34.00$

Copyright (C) 2009 by the American Society of Nuclear Cardiology. doi:10.1007/s12350-009-9125-6
Improve:

Perform correlation studies in your own lab of MPI patients who have gone on to invasive ${ }^{11}$ or noninvasive coronary angiography.

Focus-attend educational opportunities, in person and/or online, for the physician, technologist, and nursing staff.

If all these fails, what to do? One option is medical therapy. If a patient is felt to be reliable can he/she be followed medically with repeat testing in 1-2 years time? As prognosis worsens with the size and intensity of a perfusion defect, the chance of a near-term event in a patient with a borderline defect would be expected to be low. Many patients can accept some uncertainty if offered medical follow-up and a plan of action.

The contribution by Abidov offers another option, CT coronary angiography and calcium scoring (CCTA). Abidov and colleagues prospectively evaluated the efficacy of 64-slice CCTA in 199 patients following either an MPI (83\%) or stress echo (17\%) felt to be equivocal or suspected to be inaccurate (for example, a discordance between an abnormal stress electrocardiogram and normal SPECT perfusion) by the referring cardiologist. CCTA studies were considered to be abnormal if (a) a stenosis was $>50 \%$, (b) if any segment of the coronaries could not be interpreted, or (c) if the Agatson score was $\geq 400$. Cardiologists were informed of the results of the CCTA. Patients were then followed for $\geq 2$ (mean $2.3 \pm 0.7$ ) years.

The primary endpoint was the ability of a CCTA that demonstrated either normal coronaries or stenoses estimated to be $\leq 50 \%$ to identify subjects who could be then defined low risk. They defined low risk in two ways. First, among those who underwent invasive coronary angiography (ICA), that no stenoses were demonstrated on QCA during ICA that were $>50 \%$. Second, among those who did not go on to ICA, low risk was defined as the absence of the occurrence of a major adverse cardiac event (MACE). They defined MACE as cardiac death, acute MI, unstable angina, or late myocardial revascularization ( $>90$ days). They found that among 93 subjects with a normal CCTA, none underwent subsequent ICA and none of these subjects experienced a MACE. The 70 subjects in whom CCTA 
demonstrated stenoses $\leq 50 \%$ also fared well, only one had an QCA stenosis $>50 \%$ on ICA, for which the subject underwent PCI; one underwent a physiciandirected PCI despite a stenosis $<50 \%$ on CCTA and ICA; and one underwent late PCI of a lesion that progressed following the demonstration of nonobstructive disease on initial CCTA and ICA. Thus, the study demonstrated a $98-99 \%$ rate negative predictive value depending on how this is defined.

On the other hand, CCTA demonstrated a stenosis of $\geq 50 \%$ in the remaining 36 subjects (17\%). Of these, $24(67 \%)$ underwent ICA, of whom 15 had obstructive disease on ICA. All 15 underwent PCI. Of note, no patients in any of the groups experienced an MI or death during study follow-up.

Several other analyses were of note. Among the 19 subjects who underwent CCTA because of the discordance of a normal SPECT perfusion coupled with an abnormal electrocardiographic response to stress, only 1 (5\%) had a CCTA demonstrating a stenosis $>50 \%$. The authors also asked the referring cardiologists upon study entry if they would have proceeded to ICA if CCTA had not available. The cardiologists responded affirmatively in $63 \%$ of the cases yet, with the opportunity to use CCTA in this study, ultimately only $16 \%$ underwent ICA.

Multivariate analysis was also performed to evaluate the incremental value of information provided by clinical data and CCTA. The Agatston score alone added significant incremental information on top of clinical data but coronary angiography by CCTA added substantially more.

The authors are to be commended for two methodologic approaches in particular. One is their categorization of those with an Agatston score of $>400$ as having a positive CCTA. Rather than push the technique beyond its current intrinsic capabilities, this conservatism is admirable. In a previous study, Cheng et al shed light on this issue when they studied of a subgroup of patients $(\mathrm{n}=52)$ undergoing 64-slice CCTA who had Agatston scores of 300-500. Enough calcium was present in this score range that $56 \%$ of the 52 had one or more unreadable coronary segment on CT coronary angiography using a 17 -segment model. ${ }^{12}$ Second, Abidov et al's decision to include, rather than exclude, subjects in their study who had unreadable segments is also laudable.

The authors also provide a "real-world" positive predictive value (PPV) of $63 \%$ in this patient population. This compares to the higher PPV of oft-quoted studies in which "drive-by" CCTA was performed before already scheduled coronary angiography. The prevalence of obstructive CAD in this latter group is much higher than would be expected in a typical patient undergoing CCTA for clinical indications.
What problems are inherent in this potential CCTA strategy? One obvious one is that $16 \%$ of patients were exposed to three diagnostic tests each with ionizing radiation and almost all of these received further radiation when they were sent to PCI. Another is the "oculostenotic" reflex ${ }^{13}$ brought out by CCTA and ICA. In this study, by the time a subject underwent ICA, if a stenosis of $>50 \%$ was found, PCI was nearly always performed. Given the lesser risk of smaller and less intense perfusion defects, it is not clear that all of these PCI's actually benefited the subject.

The authors also focus on the coronary findings of the 199 CCTA studies and remain silent on the frequency of incidental, noncardiac findings. Fortunately, the maturation of CCTA has brought an appreciation for the increased spatial resolution observed with a smaller, more focused imaging area. This, combined with the anxiety and downstream cost created by incidental findings, has resulted in the CCTA field of view increasingly becoming the box around the heart rather than a larger view of the chest.

As discussed above, the authors found the Agatston score to aid in the prediction of obstructive disease by ICA following an inclusion MPI. On its own, the prognostic importance of calcium scoring at either low or high levels is now firmly established. Specifically adding a calcium score to an MPI, be it normal, ${ }^{14}$ equivocal or abnormal, ${ }^{15}$ has great potential to provide further incremental risk stratification. In a novel approach, Einstein et al recently demonstrated that CT attenuation maps of patients undergoing MPI on SPECT-CT or PET-CT machines can be used to visually estimate an Agatston score with reasonable accuracy without the need for an extra CT scan performed solely for calcium scoring. ${ }^{16}$

Abidov et al's contribution provides strong incremental value to the imaging community. As CCTA matures from its current teenage status to young adulthood, its potential to aid clinicians following an equivocal and discordant MPI will undoubtedly continue to mature.

\section{References}

1. Abidov A, Gallagher MJ, Chinnaiyan KM, et al. Clinical effectiveness of coronary computed tomographic angiography in the triage of patients to cardiac catheterization and revascularization after inconclusive stress testing: Results of a two-year prospective trial. J Nucl Cardiol 2009. doi:10.1007/s12350-009-9117-6.

2. Matsumoto N, Berman DS, Kavanagh PB, et al. Quantitative assessment of motion artifacts and validation of a new motioncorrection program for myocardial perfusion SPECT. J Nucl Med 2001;42(5):687-94

3. Heller GV, Bateman TM, Johnson LL, et al. Clinical value of attenuation correction in stress-only Tc-99m sestamibi SPECT imaging. J Nucl Cardiol 2004;11:273-81. 
4. Thompson RC, Heller GV, Johnson LL. Value of attenuation correction on ECG-gated SPECT myocardial perfusion imaging related to body mass index. J Nucl Cardiol 2005;12:195-202.

5. Depuey G, Berman DS, Garcia E. Cardiac SPECT imaging. Philadelphia: Lippincott-Raven; 1996.

6. Miyamoto MI, Vernotico S, Majmundar H, Thomas G. Pharmacologic stress myocardial perfusion imaging: A practical approach. J Nucl Cardiol 2007;14:250-5.

7. Iskandrian AS, Heo J, Kong B, Lyons E. Effect of exercise level on the ability of thallium-201 tomographic imaging in detecting coronary artery disease: Analysis of 461 patients. J Am Coll Cardiol 1989;14:1477-86.

8. Thomas GS, Prill NV, Majmundar H, et al. Treadmill exercise during adenosine infusion is safe, results in fewer adverse reactions, and improves myocardial perfusion image quality. J Nucl Cardiol 2000;7(5):439-46.

9. João V, Vitola JV, Brambatti JC, Caligaris F, et al. Exercise supplementation to dipyridamole prevents hypotension, improves electrocardiogram sensitivity, and increases heart-to-liver activity ratio on Tc-99m sestamibi imaging. J Nucl Cardiol 2001;8:652-9.

10. Simons M, Parker JA, Donohoe KJ, et al. The impact of clinical data on interpretation of thallium scintigrams. J Nucl Cardiol 1994;1(4):365-71.
11. Thomas GS, Wolin D. Nuclear cardiology in a managed care environment. J Nucl Cardiol 1998;5(2):210-7.

12. Cheng VY, Lepor NE, Eshaghian S, et al. Effect of coronary artery calcium score on complete coronary tree visualization by 64 -slice computed tomographic coronary angiography (abstract). Circulation 2006;114(18 Suppl II):380.

13. Topol EJ, Nissen SE. Our preoccupation with coronary luminology: The dissociation between clinical and angiographic findings in ischemic heart disease. Circulation 1995;92:233342.

14. Schwartz RG, Greenland P, Berman DS, Shaw LJ, Wilson PWF. Panel 4: evidence-based strategies for optimizing management of cardiovascular risk and therapy. In: Cerquiera M, Udelson J, eds. Seventh Nuclear Cardiology Invitational Conference, Park City, Utah, 2004. J Nucl Cardiol 2005;12:480-509.

15. Thompson RC, McGhie I, Moser KA, et al. Clinical utility of coronary calcium scoring after nonischemic myocardial perfusion imaging. J Nucl Cardiol 2005;12:392-400.

16. Einstein AJ, Johnson LL, Thompson RC, et al. Visual estimation of coronary artery calcium from low-dose CT attenuation correction scans shows close agreement with standard Agatston score in hybrid SPECT/CT and PET/CT (abstract). J Am Coll Cardiol 2009;53(10 Suppl A):A278. 